\title{
The Formation of the Sexual Nuclei in Lilium Martagon.
}

\author{
I. Oögenesis.
}

BY

ETHEL SARGANT.

With Plates XXII and XXIII.

SOME recent zoölogical observations show that in certain $S$ cases a transverse division of the chromosomes occurs during one of the karyokinetic divisions immediately preceding the formation of either sexual nucleus ${ }^{1}$. This is sufficiently remarkable, as the solitary exception to the rule of longitudinal fission of the chromosomes during karyokinesis. But it has further been shown that if future research should establish the general law that the formation of a sexual nucleus is always preceded by a transverse division of

${ }^{1}$ See the references (p. 97) in Dr. Haecker's paper: 'The Reduction of the Chromosomes in the Sexual Cells as described by Botanists'-Ann. of Bot., ix. I895.

Annals of Botany, Vol. X. No. XXXIX. September, 1896.] 
chromosomes, great light will be thrown on the relation of the process of fertilization to the phenomena of heredity ${ }^{1}$. Dr. Valentin Haecker has lately called the attention of botanists to this important subject, and has suggested that they should re-examine the critical divisions in the test case of Lilium Martagon ${ }^{2}$. A complete account of the formation of the sexual nuclei in this plant was given in I89I by M. Guignard in his well-known paper, 'Nouvelles Etudes sur la Fécondation.' The four spermatogenetic and three oögenetic divisions which follow the reduction in number of the chromosomes are minutely figured and described in this memoir. It is shown in each case that the chromosomes are divided longitudinally. Dr. Haecker has however pointed out that the first karyokinesis on either side is figured as differing somewhat from those which succeed it, and he has hinted that the cause of this distinction may be that during this nuclear division the apparently longitudinal fission of the chromosomes is in fact a transverse one. In view of the theoretical importance of such a discovery, it seemed worth while to make a detailed study of the whole series of nuclear divisions from this point of view, paying particular attention to the long period of growth and development which precedes the appearance of the reduced number of chromosomes on either side. I was encouraged to undertake this work by Dr. D. H. Scott of Kew, to whose advice and sympathy I have owed much throughout its progress. It has been entirely carried out in my laboratory at Reigate.

Before proceeding further it will be necessary to define more exactly the limits of the investigation. The genealogy of the male and female generative nuclei of any given plant of Lilium Martagon extends back through a long line of nuclear divisions to the first division of the fertilized ovum from which

${ }^{1}$ For a discussion of Dr. Haecker's hypothesis and its relation to Professor Weismann's Reduction Theory, see Rückert, Die Chromatinreduktion bei der Reifung der Sexualzellen-Ergebn. d. Anat. u. Entw. (Merkel u. Bonnet), I894.

${ }_{2}^{2}$ Vid. Haecker, 1. c. pp. 99-101. 
the plant arose. It is impossible however to distinguish those nuclear divisions which are in the direct line of descent - the 'germ track' - from those which are not, until we are within a few generations of the sexual nuclei. In the young anther as soon as the archesporial tissue is differentiated we can say with certainty that all the nuclei within it will by repeated divisions give rise to pollen mother-cell nuclei, and therefore four generations later to the male nucleus of the pollen-tube. The ancestry of the ovum cannot be traced back so far. The embryo-sac is a hypodermal cell, distinguished at first from those surrounding it by its median position only. Three successive nuclear divisions within the embryo-sac divide its primary nucleus from the ovum, and only during these three generations can the line of descent be securely followed.

Fortunately it is not necessary for our present purpose that the divisions which will form the ovum should be identified through more than three generations, nor those which are to form the male generative nucleus through more than four. For it is well known that the three karyokinetic divisions which immediately precede the formation of the ovum, and the four preceding that of the male generative nucleus, differ from their predecessors in possessing twelve chromosomes in place of twenty-four. These twelve chromosomes appear in the primary embryo-sac nucleus and in that of the pollen mother-cell respectively just before the formation of the spindle. According to Dr. Haecker's hypothesis, each of the twelve represents two of the chromosomes from the previous division joined end-to-end. The reduction in number implies no corresponding reduction in mass, for the twelve new chromosomes are in fact twelve double segments, equivalent to the twenty-four single segments of the previous karyokinesis. If the subsequent karyokineses effect a longitudinal fission of each chromatic segment as botanists have hitherto asserted, then each segment which goes to build up the nucleus of the ovum or the male generative nucleus is a descendant of one of these double chromosomes and of similar 
structure, and all the twenty-four chromosomes of the latest archesporial division are represented in the sexual nucleus. But Dr. Haecker predicts on the faith of zoölogical analogies that one of the three oögenetic nuclear divisions with twelve chromatic segments and one of the four similar spermatogenetic divisions will be found to result in the division of each segment not longitudinally but transversely, and in such a way that each half represents one of the original twenty-four chromosomes. If this should prove true, the descendants of the original twenty-four chromosomes would be divided during this karyokinesis between the daughter nuclei, twelve going to one and twelve to the other. This would be a true reduction division in Professor Weismann's sense. The sexual nucleus thus formed would be constructed of the descendants of twelve only from among the original twentyfour chromosomes.

It is clear that Dr. Haecker's hypothesis rests on three assumptions.

I. That the reduction in number of the chromosomes which has just been described is caused by the joining of twentyfour chromosomes from the previous nuclear division end-toend in twelve pairs.

2. That in a subsequent nuclear division, before the formation of the ovum on the one hand or of the male generative nucleus on the other, the twelve double segments thus formed are divided not longitudinally but transversely.

3. That this transverse fission corresponds to the junction formerly accomplished between the members of each pair.

Assumptions $I$ and 3 are not in themselves improbable, but in order to verify them it would be necessary to identify each chromosome from one karyokinesis to another throughout the intervening resting-stage. This has not hitherto been found possible. The second assumption can be proved or disproved by observation. For in so favourable a case as the anther or embryo-sac of Lilium Martagon it is very 
possible to follow three or four nuclear divisions in such detail as to place their true nature beyond a doubt. In the following pages I have tried to do this for the three critical divisions of the oögenesis : later on I hope to publish a corresponding set of observations on the four spermatogenetic divisions.

\section{BIOLOGICAL.}

The whole process of the formation of the sexual nuclei in Lilium Martagon occupies five or six weeks in May and June. At the beginning of this period, transverse sections through a number of buds from a single stalk show that the embryo-sac is differentiated in each of the median ovules of the ovary ${ }^{1}$ about the same time that the pollen mother-cells are formed in the anthers of the same flower. The process of oögenesis is finished when the ovum is formed in the embryo-sac, and this takes place as the flower opens. Perhaps a day later the male generative nucleus is formed in the tube of a pollen-grain from the same flower, so that the two processes begin and end together. Nuclear divisions in the pollen mother-cell and embryo-sac of the same flower do not seem to occur at the same time. On the contrary, an alternation of activity is to be observed (vid. Table I).

The first karyokinesis on either side is preceded by a period of growth and development which may be described under two heads.

I. Nucleus in resting condition: it increases in size without material alteration in structure.

2. Process of formation of spirem thread within nucleus, including the stage called synapsis.

1 The upper and lower ovules in each ovary are always smaller than those in the middle, and usually in an earlier stage of development. The stage of the median ovules is throughout considered typical of that of the ovary. 
TABLE I.

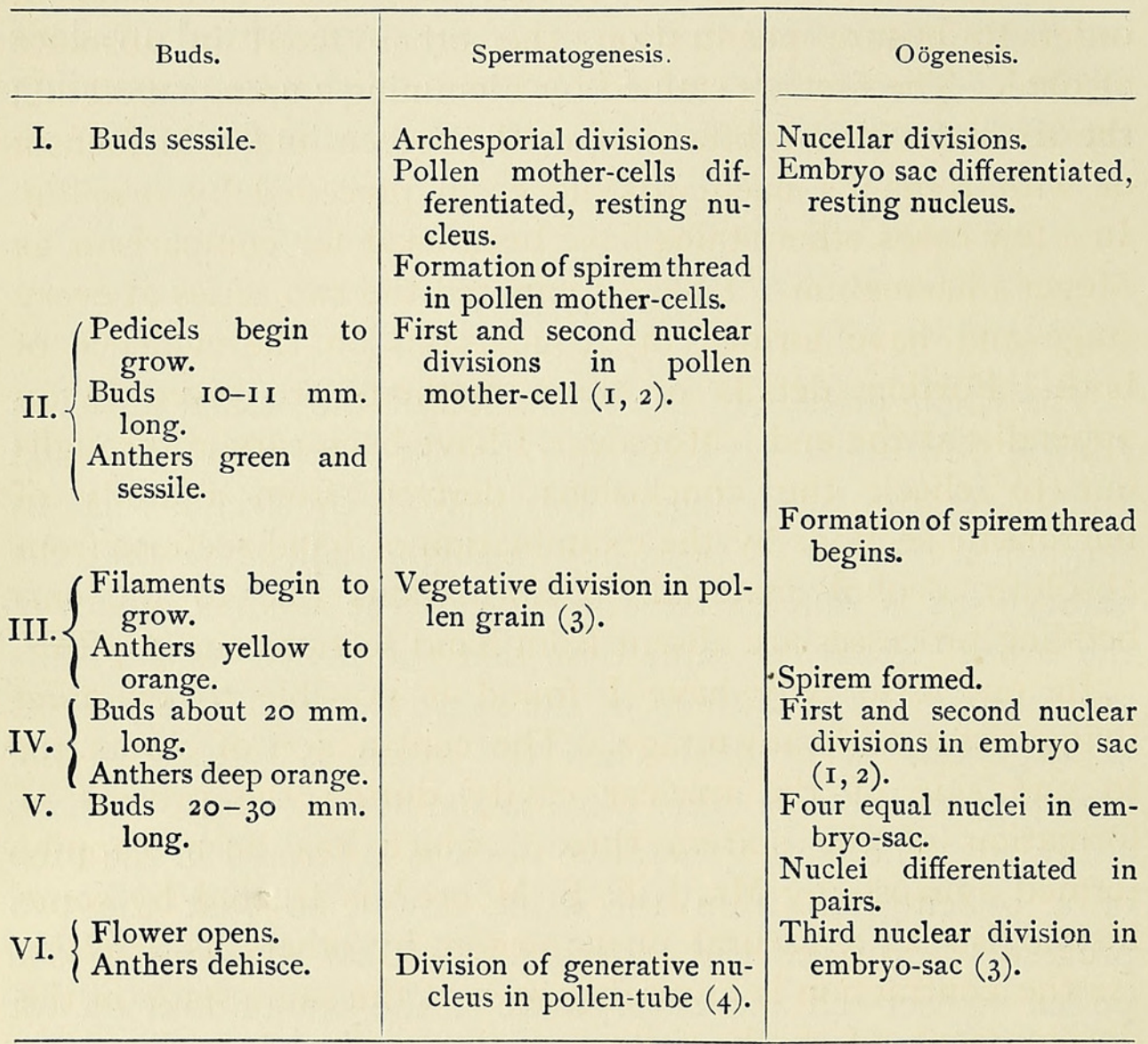

\section{METHODS.}

The methods of the histologist are necessarily indirect. $\mathrm{He}$ is seldom able to observe the structures he describes while they are alive. His only resource is to arrest their movement by killing them as instantaneously as possible, and from a series of the figures thus obtained to attempt a reconstruction of the process of growth. It is therefore essential to bear in mind the possibility of errors arising ( 1 ) from deformation produced by the processes of preparation, and (2) from faulty seriation.

Owing to the mass of material dealt with, I have found it impracticable to use consistently more than two fixing methods. My preparations however are in two paralle] series 
throughout: one set fixed in one of the osmic acid mixtures, sometimes made up with a certain proportion of alcohol in order to ensure penetration, the other fixed in absolute alcohol. The first are stained by Flemming's orange method; the second with a mixture of methyl-green and acid fuchsin or with Renaut's haematoxylic eosin preceded by orseillin. In a few cases other stains have been used for comparison, as Meyer's haemalum. I have compared the two series at every stage and have formed my conclusions on the evidence of both. Further details of these methods are given in an appendix at the end. Moreover, I have been careful throughout to check the conclusions derived from a study of microtome sections by the examination of hand sections from absolute alcohol material. Deformations due to the embedding processes are absent from hand sections cut in pith.

In one case only have I found it possible to examine living tissue with advantage. The contraction of chromatin to one side of the nuclear cavity during the process of formation of the spirem thread, which has been happily termed synapsis by Mr. J. E. S. Moore ${ }^{1}$, is treated by some histologists as a natural phenomenon, by others as artefact. As the contraction is invariably seen at the same stage in the development of both pollen mother-cell and embryo-sac nuclei, however prepared, I have for some time considered it as a condition existing during life, though doubtless subject to deformation during fixing and cutting processes. This summer however I obtained sections from fresh anthers on several occasions in which the chromatin of the pollen mother-cell nucleus could be clearly seen collected into a ball on one side of the nuclear cavity. The chromatin was identified by subsequent fixing and staining of the section.

\section{Division of the Vegetative Nucleus.}

The nuclei with which we are concerned in this paper are, as already stated, peculiar in exhibiting twelve chromatic 
segments during karyokinesis in place of the usual twentyfour chromosomes. It is essential that any other deviations from the normal type of karyokinesis should be carefully observed and recorded, that they may be fully accounted for and the possibility of their masking a transverse division considered. For this purpose comparison with the details of nuclear division in the vegetative tissues of Lilium Martagon is needed. Figs. I to 8 represent nuclei belonging to the integuments of the ovule. In the sections from which they are taken the embryo-sac has nearly attained its full size: there can be no doubt therefore that these nuclei are off the 'germ track.' Owing to the flattened shape of the cells, the karyokinetic figures are apt to be spread out in a manner convenient for counting the chromosomes (vid. Figs. 3 and 4). They are also of comparatively large size. I have compared other nuclei from the tissues of anthers and ovaries with these and find that the details of division are identical. When the cell is cylindrical the spindle is not flattened, and then resembles Fig. 9, but the separation of the chromatic segments takes place in just the same way.

The structure of the resting nucleus is most clearly made out in tissue fixed with Flemming's solution and stained by his orange method (Fig. I). Within the nuclear membrane a network of fine dotted threads is partially masked by cloudy masses of granular substance. In alcohol preparations stained with methyl green and acid fuchsin this substance is bright green. The threads indeed in such a preparation are not visible at all, but the cloudy green substance is seen to be disposed in a coarse mesh-work. Returning to the Flemming preparations, it is a question whether the threads really anastomose with each other. They appear to do so, and there is always a comparatively large dark dot at the junction of two or more threads, but it is possible that this is an optical effect and that the threads only pass very closely behind each other. Thus the possibility of the whole network being in fact composed of one continuous much-tangled filament is not excluded. The whole nuclear cavity is 
traversed by these threads, but the patches of cloudy substance, which I shall call amorphous chromatin, seem more numerous towards the periphery. In the larger meshes of the network are several spherical nucleoli of various sizes.

The first stage which can be identified as indicating approaching division is the slender spirem (Fig. 2). The nuclei are decidedly larger than in the resting stage and their structure is very different. The nuclear membrane is still distinct, and within it is a single slender ribbon much convoluted and enclosing within its curves several small nucleoli. No anastomoses are seen, and the ribbon takes a uniform green colour in methyl green. The amorphous chromatin has entirely disappeared. No doubt it has served to feed the ribbon, but I have not been able to find a series of intermediate forms which would link the structure of the resting nucleus to that of the slender spirem. Transitional stages between slender spirem and early spindle (Fig. 4) are easily found. The ribbon becomes shorter and broader so that the convolutions are more simple. At last the nuclear membrane vanishes and the nucleoli disappear. The ribbon then falls into segments. In the stages immediately preceding this the ribbon shows signs of a certain method in its twistings. I have never been able to follow it throughout its course on account of the small size of the nucleus and the number of coils within it. But the general impression left after examining many figures is that there is a definite arrangement of coils resembling that figured by Professor Flemming for the spermocytes of Salamandra ${ }^{1}$.

Figures such as that shown in Fig. 4 are extremely common, and in the absence of any direct evidence concerning seriation I was at first inclined to consider them as late stages in which the segments pointing upwards were about to proceed to one pole, and those pointing downwards to the other. On being counted, however, the chromatic segments turned out to be about twenty-four in number, whereas

1 W. Flemming, Neue Beiträge zur Kenntniss der Zelle, Pl. xxiii. fig. 2. 
if the fission had already taken place they should have been about forty-eight ${ }^{1}$. The discovery of earlier intermediate forms, such as that drawn in Fig. 3, and of rather later figures in which the chromosomes though still directed towards either pole show clear longitudinal fission (Fig. 5), confirmed the conclusion that the stage in question (Fig. 4) occurs before the formation of a typical nuclear plate. The ribbon-like character of the segments in Figs. 3 and 4 is marked.

The chromosomes when arranged to form the nuclear plate (Fig. 6) lie in a plane perpendicular to the axis of the spindle. The separation of their segments has begun. Bundles of spindle fibres are attached to each segment near one end, and the segments separate exactly as if they were pulled apart by ropes fixed at this point (Figs. 6 and 7). Each segment as it nears the pole is seen to be hook-shaped (Fig. 7). In Fig. 8 the daughter nuclei are seen to be fringed by the longer legs of the hooks not yet drawn up into them.

The daughter nuclei at first consist of naked coils of chromatic ribbon. By degrees the coils open, a nuclear membrane is formed, nucleoli appear, and the chromatic ribbon lengthens. The nuclei ultimately pass into the restingstage by a series of changes which I am unable to decipher. The cell-plate and cell-wall are formed in the usual way.

\section{OÖGENESIS.}

While the nucellus is still a mere hump on the placenta, its tissues contain numerous dividing nuclei. The nucleus of any hypodermal cell occupying a fairly median position may be the ancestor of a future embryo-sac nucleus. All these divisions however conform strictly to the vegetative type. The spindle figures differ somewhat in shape, in some cases being equally developed all round the axis of the spindle (Fig. 9), in others flattened as is usual among integument nuclei (Fig. 4). In one such case I was able to count

${ }^{1}$ It is extremely difficult to count the chromosomes is a small spindle figure with perfect accuracy. I have uniformly found about twenty-four chromosomes in nuclei from vegetative tissues of Lilium Martagon. 
the chromosomes accurately and found twenty-four of them. In all others difficulties of observation prevented me from determining the number with absolute certainty. The question always lay between twenty-three and twenty-four, or between twenty-four and twenty-five.

A median hypodermal cell becomes larger than its fellows some time before the appearance of either integument. The nucleus of this larger cell usually remains for some time in the resting-stage and becomes the embryo-sac nucleus without further change, but not infrequently it divides once again. This accounts for the occasional appearance of twin embryosacs which is not infrequent in very young stages. In other cases two nuclei are found in the same embryo-sac long before the regular period for division, and I have seen several instances of twin spindles. One preparation shows a double embryo-sac with four spindles in it which is evidently a case of a twin second division. These anomalous cases are not numerous enough however to account for all the instances in which I have found a nuclear division taking place within an enlarged hypodermal cell. Probably the rule is that one of the daughter-cells increases in size at the expense of the other and becomes the embryo-sac. The karyokinesis itself is of the usual nucellar type.

The structure of the resting nucleus in the very young embryo-sac is precisely similar to that of the nucellar nuclei surrounding it. A network of dotted threads is partially concealed by the substance I have called amorphous chromatin. There is either a single large nucleolus, or more commonly several of different sizes (Figs. Io and Io $a$ ). A glance at Table I will show that the nucleus remains in the resting state until the pollen mother-cells have completed their double division. This probably takes about a fortnight. During this time the nucleus increases in size, almost doubling its diameter, without materially altering in structure (Fig. I I). The threads become thicker and their dots larger, but the latter still form a single row (Fig. I I $a$ ). Anastomoses seem to take place, but may be only apparent. The amorphous 
chromatin is very much less conspicuous : instead of a heavy deposit of cloudy substance which in methyl-green preparations is of a bright colour and completely masks the thread over which it lies, we have a scanty grey-green precipitate which makes the thread look ragged. This disappearance of amorphous chromatin is partly accounted for by the increase in size of the nucleus, for the area of each nuclear section is nearly four times as great as it was, but there can be little doubt that there has also been a real decrease in quantity of this substance.

If we refer back to the series of preparations bridging this interval, we find that as the nucleus increases in size the network of dotted threads begins to occupy a peripheral position and the amorphous chromatin loses its cloudy look and is heaped more closely over the threads, the meshes being left clear. It is difficult to avoid the suspicion that the chromatin, which was at first in the cloudy probably dilute state which I have described, is now being deposited on the dots in the network.

The nucleoli of the later resting-stage are still spherical and of well-defined outline, but they often look swollen. Careful examination of the chromatic network at this stage reveals drops adhering to it which are rounder and more regular in outline than the fragments of amorphous chromatin. In methyl-green and fuchsin preparations the larger of these dots are distinctly red, and they must therefore be considered nucleolar. These are the first indications of the solution of the nucleolus which follows during synapsis.

The transition between the late resting-stage just described and the contracted state known as synapsis is very instructive, and is marked by ( $\mathrm{I}$ ) the increase in thickness of the chromatic thread and the appearance in it of a double row of dots; $(2)$ the gradual solution of the nucleolus; and (3) the partial disappearance of the nuclear membrane. In the earlier transition stages the nucleolus is still more or less spherical and clearly outlined, but the number of small drops of nucleolar matter ( $n$, Fig. I $2 a)$ attached to the thread 
has very much increased. Perhaps they may be derived from the breaking down of the smaller nucleoli. The thread itself is much thickened, often irregularly: in many places the drops of nucleolar matter adhering to it have elongated in the direction of the thread. This suggests at once that the nucleolar matter is applied to the thread with the purpose of thickening it. Where the thread is of fairly uniform thickness for some distance, two rows of dots can sometimes be made out in it with very high powers. The picture is usually confused by the twisting on itself of the thickened and rather flat thread (Fig. I 2 a). Where the double row can be made out with any precision, which at this stage is only in preparations from Flemming material stained by the orange method, the ribbon itself is seen to be of a reddish tinge and the dark dots to border it on either side. The nucleolus usually becomes vacuolated before losing its clear outline.

Before finally passing into the contracted state the dotted ribbon just described begins to close round the nucleolus. This has become washy and vacuolated. Large fragments are broken from it: some of these, or the main nucleolus itself, are often sickle-shaped. Smaller fragments are attached to the chromatic ribbon. The outline of the nuclear membrane can hardly be traced.

The nucleus now enters the period of synapsis, in which it remains for some time, probably several days. The whole chromatic thread contracts into a ball, leaving a large part of the nuclear cavity empty. This cavity is somewhat swollen: the membrane can be followed in places, but is often broken and always obscure (Fig. I3). The chromatic ball is always close to one side of the nuclear cavity, and partly surrounds the half-dissolved nucleolus, which is often sickle-shaped (Fig. 13). A general view of the whole structure is best gained from a thick alcohol section stained with methyl-green and fuchsin, as the nucleolar matter is then sharply distinguished from the chromatin. Microtome sections are unsatisfactory, as the chromatic thread as well 
as the nucleolus is often displaced and broken by the knife. Tangential microtome sections however give details of the structure of the chromatic thread very well in places.

Two periods of synapsis may be distinguished. During the first the chromatic ribbon grows greatly in width but the dots continue small. (Cf. Fig. 1 $3 a$ with Fig. 13 $b$ : 13 $a$ is drawn from a nucleus which has just entered the first period of synapsis, and $\mathrm{I}_{3} b$ from the nucleus figured at $\mathrm{I}_{3}$ which is near the end of the same period.) This early period is marked by a large and washy nucleolus of irregular outline and by the ill-defined nuclear membrane. In the second period the nucleolus is well defined and spherical, and the nuclear membrane reappears. The ribbon does not become wider, but the dots which border it increase in size. The coils of ribbon also become looser and occupy a larger part of the nuclear cavity. Insensibly the structure of the nucleus becomes that of the well-known spirem stage (Fig. I4. Cf. also Guignard's Figures 44-47, 1.c.).

We have now traced the formation of the spirem thread in great detail. The linin ribbon bordered on either margin by a row of chromatin granules can be followed back to the beginning of synapsis. There can be little doubt that this double row is formed just before contraction of the chromatic thread by the fission of the pre-existing single row (cf. Figs. I I and i2). The linin ribbon at this period and during early synapsis seems to be fed from the partially dissolved nucleolus. I am inclined to think that the solution of the nucleolus is brought about by the entrance into the nuclear cavity of some liquid from the cytoplasm, otherwise it is difficult to explain the temporary disappearance of the nuclear membrane. The contraction of the chromatic coils round the nucleolus is possibly in order that the interstices may be too small to admit of the escape of semi-fluid nucleolar matter. We shall meet with some of these phenomena again at a later stage.

The structure of the nucleus in the spirem stage is too well known to need a long description. In hand sections 
from alcohol material I have never found free ends of chromatic ribbon except where the nucleus is touched by the knife ${ }^{1}$. Nor are any anastomoses visible. The ribbon seems to consist of a single coiled and twisted erythrophilous filament, bordered on either margin by a row of cyanophilous dots. Microtome sections about $10 \mu$ thick from Flemming material show structural details of the ribbon very clearly, and it is possible, by careful comparison with the much thicker hand sections, to make sure that we are dealing with a true spirem stage.

We are now approaching the point in the development of the embryo-sac nucleus at which the reduced number of chromatic segments first appears. The history of their development from the single ribbon of the spirem stage is undoubtedly the most important passage in the whole investigation. In order to appreciate the evidence it is necessary to anticipate a little and examine the structure of the mature chromosome ${ }^{2}$. Fig. 20 represents several chromosomes lying loose in the nuclear cavity before the appearance of a spindle. Each is composed of two segments which are twisted round each other. In some cases it is clear that these segments are quite distinct; in others they seem to be joined at one end. It is impossible however to be certain that this appearance is not due to the close approximation of two free ends. At an earlier stage (Fig. 19) the chromosomes are longer, and each segment is an erythrophilous ribbon bordered on either margin by a row of cyanophilous dots. The figure is drawn from a median section in which the chromosomes are clustered round the nucleolus in a very characteristic way (Fig. I9). The ribbon-

1 The preparation from which Fig. I4 was drawn for instance is not quite perfect. Four free ends are seen in the lower left-hand region, but these all lie in the same plane at the upper surface of the nucleus, and are therefore obviously due to the removal of a tangential section from that surface.

${ }^{2}$ As a matter of convenience I shall speak of the twelve chromatic segments which take part in the first karyokinesis of the embryo-sac nucleus as chromosomes. In doing so I do not mean to express any opinion as to their relation to the twentyfour chromosomes of the previous division. 
like character of each segment and the twist of the two segments of each chromosome round each other are even clearer in tangential sections of nuclei at this stage. Such sections often show the structure of one or two chromosomes with diagrammatic precision (Fig. I9 $a$ ).

The question to be resolved by the examination of stages intermediate between that just described (Fig. I9) and the typical spirem (Fig. I4) is of fundamental importance. The structure of each ribbon-like segment in the immature chromosome precisely resembles that of the spirem ribbon itself. Suppose the spirem ribbon to have fallen into twelve lengths, each of which then doubled on itself, the free ends twisting round each other. The transition to such forms of chromosome as those represented in Figs. I9 and 20 would be accomplished by simple contraction of the twisted lengths of ribbon, together with a fission at the sharp bend in some cases. The fate of the twisted segments can be followed with perfect certainty (Figs. 20-24). During karyokinesis each pair is separated, untwisting during the process, and thus half the segments go to one pole, half to the other. If the two segments of each chromosome had been originally one long length of spirem ribbon, the karyokinesis separating those segments would in fact divide each chromosome transversely.

The process of formation of twelve chromosomes from the continuous spirem ribbon can be followed only in sections thick enough to include the whole nucleus. I have therefore worked out the earlier stages entirely by means of hand sections from alcohol material. Figs. 14-18 are drawn from such preparations, and we are at once struck by the fact that the spirem ribbon begins to split longitudinally while it is still continuous (Fig. I5). It is not separated into lengths until this longitudinal fission is complete, and each length therefore consists of two distinct filaments twisted round each other and not of a single comparatively broad ribbon (Fig. 16). Each filament is necessarily formed of a row of chromatin granules connected by a linin thread.

The double filaments begin at once to contract, and while 
doing so they cluster together at one side of the nuclear cavity round the half-dissolved nucleolus (Figs. I7, I8). The nuclear membrane at the same time becomes indistinct. All the phenomena of synapsis have in fact reappeared. After contraction has proceeded for some time each filament shows a double, not a single row of dots. The stage at which this can be clearly made out depends a good deal upon the preparation. That from which Fig. I 8 is drawn, for instance, suggests a double row on careful examination, but the thickness of the section prevents absolute certainty. At a rather later stage the double row of dots can be quite clearly seen in microtome sections. I am inclined to believe that the actual fission of the original single row of granules in each filament takes place very shortly after the longitudinal splitting of the spirem ribbon. This is the second occasion on which we have found the phenomena of synapsis associated with the fission of a row of chromatin granules.

The double filaments may now be called chromosomes. They continue to shorten and broaden, and soon acquire the appearance represented in Fig. I9 $a$. Contraction indeed continues up to maturity (cf. Fig. 20 with Fig. I9), but shortly before that time a curious change in the structure of the chromosome takes place. Chromatic dots can no longer be distinguished on segments of linin ribbon. The whole chromosome stains uniformly like chromatin. I cannot even make out whether a fission in each mature segment corresponds to the interval between the two rows of chromatin granules. Each segment looks perfectly homogeneous.

One effect of this change is to make it much more difficult to distinguish between the segments of a single chromosome. The twist of the segments on each other naturally becomes closer during contraction, but while the outline of each was traced by a row of dark dots the compound character of the chromosome was clear. After the segments have become uniform in colour it is only in favourable cases that their twist on each other can be made out at all (Figs. 20, 2I).

Little remains to be said as to the behaviour of the 
chromosomes during karyokinesis. Traces of their grouping round the nucleolus persist until the nucleolus and nuclear membrane disappear together (Fig. 20). The spindle appears at first as an irregular sheaf of fibres which quickly becomes symmetrical. Figs. $2 \mathrm{I}$ and $22(a-c)$ show how the chromosomes are attached to the bundles of spindle fibres. The point of attachment is sometimes about the middle of the chromosome, sometimes near one end ${ }^{1}$. The segments untwist from each other as they are pulled apart. This probably accounts for their strained and knotted look just before separation (Fig. 23). In the diaster (Fig. 24) the segments are generally $\mathrm{V}$-shaped, their angles directed towards either pole ${ }^{2}$.

The large size of the spindles and the reduced number of chromosomes makes it fairly easy to count the latter in thick sections. Ambiguity often arises however from the different ways in which the chromosomes separate. It is sometimes impossible to distinguish between a single chromosome separating in the middle and two adjacent end-splitting ones. In thin serial sections one or more chromosomes are commonly shattered by the knife, and the fragments can seldom be pieced together with certainty. Nevertheless, I was able in fourteen cases to count twelve chromosomes in the nuclear plate. In many other instances from among some hundred spindles the question lay between eleven or twelve chromosomes, and in a few it was doubtful whether there were twelve or thirteen. I have no instance of a number so low as ten or so high as fourteen being even suggested.

The daughter nuclei first appear as two tight knots of chromatic ribbon. Their construction from the diaster segments cannot be followed. Spherical drops of erythrophilous substance commonly appear in the cytoplasm about this

1 A typical example of a chromosome separating from the middle is shown in Fig. 21. It is instructive to compare it with a similar chromosome applied to the nucleolus in Fig. 20. This suggests that nucleolar matter serves, if not as material for spindle fibres, at least to solder them to chromosomes.

2 I have one preparation in which the diaster segments are hooked as in the vegetative nucleus. 
time. Later on, as the coils of the daughter nuclei open out, the number of drops increases, and some are found inside the nuclei (Fig. 25). There can be little doubt that we here have drops of nucleolar substance from which the new nucleoli are being reconstructed. The coils of chromatic ribbon are at first coloured uniformly like chromatin, but later on they show the familiar structure of a linin ribbon bordered with dark dots (Fig. 25). No doubt the dots are granules of chromatin, but their identity with those bordering each segment of the immature chromosome (Fig. 19 a) cannot be traced. The cell-plate is still clear between the nuclei. It finally disappears when they have passed into the restingstage (Fig. 26).

We may now sum up the conclusions drawn from our examination of the first division of the embryo-sac nucleus. The chromosomes have been identified with lengths of the ribbon so clearly shown in the well-known spirem stage, Each segment of a single chromosome represents one of the two parallel rows of granules found in such a length of ribbon. It has never been doubted that these arose by fission of a single row at an earlier stage ${ }^{1}$, and the history of their development confirms this view. The karyokinesis therefore which separates the two segments of each chromosome from each other does in fact divide the chromosomes longitudinally.

The history of the second longitudinal fission - that which takes place within each segment-is not so satisfactorily completed. It is not certain that this fission persists in the mature chromosome (vid. ante, p. 46I). Even supposing that it does, and that the chromatin granules mentioned as appearing in the dispirem can be identified with the granules of the immature chromosome, all trace of fission is again lost in the resting-stage of the daughter nuclei. It is not impossible indeed that the slender thread of this stage bears a double row of granules, but there is no evidence that it does so. The existence of four rows of granules in the immature 
chromosome however is interesting, since it recalls the structure of the corresponding stage in the spermocyte of Ascaris megalocephala. Here also Dr. Brauer has traced the four rows to a double longitudinal fission ${ }^{1}$.

The two nuclei which now occupy the embryo-sac (Fig. 26) soon show signs of approaching division. They increase in size, and the ragged-looking network characteristic of the resting state gives place to a neat spirem ribbon. Some preparations show this ribbon clearly bordered on either side by a row of dark dots. It very soon becomes of a uniform colour. At first both nuclei possess a nuclear membrane and nucleoli, and even at this early stage a difference in size between them may often be observed (Fig. 27). The chalazal nucleus is commonly larger than that at the micropylar end. Both nuclei are sometimes surrounded by a comparatively clear space divided from the cytoplasm by a granular boundary (Fig. 27). After disappearance of the nuclear wall this clearer space persists, and well-marked radiations are found in it. A washy nucleolus can still be perceived among the tangled chromatic coils (Fig. 28).

Segmentation of the naked spirem ribbon takes place while it is still much tangled and of irregular breadth (Fig. 29). The spindle-fibres have appeared and seem to stretch the figure in one direction, so that the young chromosome is much pulled out in that part which lies along the spindlefibres and twisted or knotted at the free ends. By degrees the chromosomes contract to uniform breadth, though their ribbon-like character is still apparent. The spindle has also become better defined and more regular, and each chromosome lies on it with one end in the equatorial plane, the other extended to one of the poles.

The chromosomes can now be counted at least roughly, and the well-known distinction between the nuclei becomes apparent. There are about twelve chromosomes in the micropylar nucleus, but commonly not less than twenty-four in the

B Brauer, Zur Kenntniss der Spermatogenese von Ascaris megalocephala (Fig. 25). 


\section{Sexual Nuclei in Lilium Martagon.}

chalazal one. I have not been able to trace any difference beyond that of size between the two nuclei during segmentation. The chromosomes when fully formed are quite as large in the chalazal as in the micropylar nucleus. Their number can be determined most easily somewhat later when the nuclear plate is formed. Exact results however are rarely to be obtained on account of difficulties of the same kind as those experienced in the previous division. They are increased in this case by the small size of the spindles and slender shape of the chromosomes. I have found but two micropylar spindles in which there could be no doubt about the number of chromosomes. In each case it was twelve. Twenty other micropylar nuclei allowed of approximate counting. In sixteen there were either eleven or twelve chromosomes, and in two either twelve or thirteen chromosomes. Of the two remaining nuclei one possessed either eleven, twelve, or thirteen, and one either ten, eleven, or twelve chromosomes. The exact number of the chromosomes in the chalazal nucleus is of less importance, and the results much more uncertain on account of the crowded nuclear plate. Among twenty-five spindles in which the chromosomes could be approximately counted, one had about twenty, nine about twenty-four, five about twenty-eight, and ten about thirty-two chromosomes.

Our object being to trace the formation of the ovum, we are strictly concerned with the micropylar nucleus only, and Figs. 29-3I are drawn from it. The chalazal nucleus however goes through the same changes at the same time; indeed, the correspondence between the two nuclei is very striking. They are always in exactly the same stage of division.

The chromosomes gradually move into an equatorial plane to form the nuclear plate, but before this is accomplished the longitudinal fission of each can sometimes be observed. The separation of the segments takes place exactly as in the vegetative nucleus (cf. Figs. 30 and $3 \mathrm{I}$ with Figs. 6 and 7). Occasionally however a chromosome begins to open about the middle of its length ( $x$, Fig. 30 ), just as those of the 
previous karyokinesis so frequently do. The diaster segments are commonly hooked, but occasionally a V-shape is found ( $\mathrm{x}$, Fig. $3 \mathrm{I}$ ). The reconstruction of the daughter-nuclei goes on in the same way as after the first karyokinesis (cf. Fig. 33 with Fig. 25). The pairs of nuclei may differ greatly in size (Fig. 32) or be nearly equal (Fig. 33). This seems to depend somewhat on the size of the embryo-sac. When it is comparatively long at the time the second karyokinesis takes place, the two dividing nuclei are widely separated, and the micropylar nucleus is commonly much smaller than the chalazal one. This difference is shown most clearly during the later stages of division. By the time the daughter nuclei have reached the resting-stage they are already differentiated in pairs. The two egg-shaped nuclei at the micropylar end are cut off from the larger flattened chalazal nuclei by the formation of vacuoles as the embryosac lengthens, and no further change takes place before the third karyokinesis (cf. Fig. 37). In short embryo-sacs the two dividing nuclei are often of equal size (Fig. 33), and the four resting nuclei are similar and placed at nearly equal intervals from each other (Fig. 34). The increase in length of the embryo-sac then takes place later.

In such embryo-sacs as these I have found some curious stages before differentiation of the nuclei in pairs. They are too numerous to be treated as pathological. The four similar nuclei are found in all the early stages of division (Figs. 35, 36 ), but they never reach the stage of regular spindle formation, though I have twice seen indications of spindle-fibres surrounding the well-differentiated naked spirem. These spirem figures are always arranged in the same way within the embryo-sac-a single nucleus near the micropyle and a group of three at the chalazal end. I have never found embryo-sacs with eight nuclei in the neighbourhood of these ovules, and do not believe that the division thus indicated comes to anything. As serial sections from older ovaries show no abortive or unhealthy ovules, I suppose that the four nuclei after making this false start return to the resting- 
stage, and become differentiated in pairs in the usual way (Fig. 37).

Between the end of the second karyokinesis and the beginning of the third, some time elapses (vid. Table I), during which the embryo-sac increases in length. Vacuoles are formed which divide the pairs of nuclei from each other. The two micropylar nuclei continue egg-shaped and small: the lower ones are larger and of less regular shape. Very often the upper chalazal nucleus is flattened as if pressed between the lower one and a vacuole, while the lower chalazal nucleus is of a shape determined by that of the end of the embryo-sac into which it fits (Fig. 37). I have described elsewhere the curious difference apparent between the lower chalazal nucleus and the other three nuclei during the third karyokinesis in the embryo- $\mathrm{sac}^{1}$. It is conspicuous as soon as the nuclear division begins. While the spirem ribbon in the three upper nuclei is beautifully differentiated, the lowest nucleus, though swollen, is still in the resting condition (Fig. $3^{8}$ ). It then proceeds to divide by the direct or amitotic method (Figs. 39-4I). The chromatin aggregates at either end as the nucleus lengthens, and the linin network between the masses of chromatin is drawn out in threads. In the later stages of division these give the figure a strong resemblance to a badly preserved dispirem (Fig. 4I). The nucleolus disappears during the process: sometimes a washy mass of semifluid nucleolar matter can be seen outside the nucleus (n, Fig. 39).

Meanwhile the karyokinetic division of the three upper nuclei goes on quite typically. We are chiefly concerned with the lower micropylar nucleus which will give rise by its division to the ovum and to the upper polar nucleus. The chromosomes however divide and their segments separate in exactly the same manner in all three figures. The process is also simultaneous, so that if one section of a series shows a single spindle in a particular stage, we are certain to find two others in neighbouring sections at exactly the same point

${ }^{1}$ E. Sargant, on direct Nuclear Division in the Embryo Sac of Lilium Martagon-Ann. of Bot., x. I896, p. I०7. 
of development. Segmentation takes place just as in the second karyokinesis. The chromosomes are at first twisted, but become straighter and arrange themselves in the usual way so as to mask the spindle (cf. upper nucleus in Figs. 39, 40). Longitudinal fission of each chromosome appears about the time that the nuclear plate is formed, and separation of the segments follows according to the vegetative type (Figs. $42,43)$. Occasionally a chromosome is found in which separation has begun near the middle, but this is not so common as during the corresponding period of the second karyokinesis.

As usual, only a small proportion of the whole number of preparations have spindle figures sufficiently clear and perfect for satisfactory counting of the chromosomes. And as I have not so many preparations of the third nuclear division in the embryo-sac as of the two previous ones, the whole number of countings is not so great. One spindle figure of each micropylar nucleus shows twelve chromosomes quite clearly. In seven other cases there are about twelve chromosomes in the nuclear plate of each. Among twelve comparatively clear chalazal spindles, five have about twenty-four chromosomes, and seven a larger number, not exceeding thirty-four in any case.

Reconstruction of the daughter nuclei proceeds as after previous divisions. Four nuclei are in a group near the micropyle, four at the chalazal end. One of the micropylar group is the nucleus of the ovum. With its formation the study of the oögenetic nuclear divisions ends.

\section{ConCLUSions.}

As regards the main object of this investigation the conclusion is clear. The chromosomes are divided longitudinally during each of the three nuclear divisions which precede the formation of the ovum. The first of these divisions does in fact differ considerably from the other two, which follow the vegetative type of karyokinesis, but this difference cannot be interpreted as concealing a transverse fission of the chromosomes. I am at a loss to explain the meaning of this 
deviation from the ordinary type of karyokinesis, but its nature and extent are worth consideration.

The characteristic features of the first division of the embryo-sac nucleus are (I) the long period of growth and development before the formation of the spirem thread and its division into chromosomes, and (2) certain peculiarities of form which characterize the chromosomes while separation of the segments is taking place. To this variation in shape of the chromosomes little importance need perhaps be attached. They are not alike when the spindle first appears. Each is formed of two segments much twisted on each other, but the number of twists, their tightness, and the disposition of the free ends vary in different chromosomes. The spindle fibres commonly attach themselves to one of the loops in the double chromosome, or sometimes to one end. It is clear that the two segments of a chromosome will not separate in the same way when they are pulled apart from the middle as when the loop where separation begins is near one end or the spindle fibres are fixed to the ends themselves. Besides this, it must be considered that the segments of each chromosome untwist from each other during the process of separation, and this of itself gives rise to much variation in shape, and often causes two adjacent chromosomes in the same nuclear plate to look very different (cf. Fig. 22, $a-c$ ). The segments of a chromosome during karyokinesis of the vegetative type separate regularly from one end and on one uniform plan, probably because they are little if at all twisted on each other (Fig. 5), and do not appear until the chromosomes are in order on the spindle. Thus the peculiarities of the mature chromosomes in the first division of the embryo-sac nucleus can be traced back to early stages in its development.

The history of that development has already been told in detail.

The nucleus passes through four phases - resting-stage, synapsis, spirem, and segmentation. It is interesting to enquire whether parallels can be found to any or all of these stages in the history of the vegetative nucleus. 
I. The resting-stage is the same in both. The young resting nucleus of the embryo-sac cannot be distinguished from the nucellar nuclei which surround it. Later on it surpasses them in size, but in structure is essentially the same.

2. The condition of synapsis is peculiar to the primary embryo-sac nucleus, and to that of the pollen mother-cell. Its characteristic features are contraction of the chromatic thread to one side of the nuclear cavity, partial solution of the nucleolus, and partial disappearance of the nuclear membrane. I have never met with a similar contraction either in vegetative nuclei or in those produced by embryo-sac divisions. Partial solution of the nucleolus may sometimes be observed in the four nuclei of the embryo-sac about the stage of Fig. 34. Possibly this may be an indication of approaching division. It should be observed however that the contraction, which is by far the most striking feature of synapsis, may perhaps be of little importance compared to the other characters. If, for example, it were merely a device to prevent the half-dissolved nucleolar matter from escaping into the cytoplasm, it would be useless in a smaller nucleus, for the meshes of the chromatic thread would be fine enough to serve this purpose without contraction. Partial solution of the nucleolus together with a vaguely outlined membrane are characters which in such a nucleus would readily escape observation.

3. The structure of the spirem stage in the embryo-sac nucleus is very different from that of the vegetative spirem. Perhaps they should be distinguished by different names. They both possess a coiled chromatic ribbon and nucleoli within the nuclear membrane. The ribbon of the vegetative spirem however stains uniformly like chromatin, while the spirem ribbon of the primary embryo-sac nucleus is erythrophilous, and bordered on either margin by chromatin granules. I have searched in vain among vegetative nuclei for a slender spirem in which a similar differentiation may be observed. It is very possible however that such a stage may exist while 
the spirem ribbon is still so narrow that in such small nuclei as we are now considering the double row of dots cannot be distinguished from a single row. I am the more inclined to believe it, as the double row of dots is found in the spirem stage of the second and third embryo-sac divisions, which in all other respects conform to the vegetative type (ante, p. 464). Supposing that this is the case, the difference between the vegetative spirem and that of the first embryo-sac nucleus would amount to this, that the change from a dotted to a homogeneous ribbon occurs early in the history of the vegetative spirem, while in the primary embryo-sac nucleus it is postponed until after segmentation has taken place.

4. Segmentation of the spirem ribbon, that is its division into lengths by transverse fission, occurs in every karyokinesis. In that of the primary embryo-sac nucleus it is preceded by longitudinal fission of the whole spirem ribbon, whereas in the vegetative nucleus no traces of longitudinal fission can be perceived until the chromosomes are in position on the spindle.

On the whole, we may conclude that the real departures from the vegetative type of karyokinesis shown by the primary embryo-sac nucleus occur during the earlier stages of development. They can only be partially referred to our ignorance of those stages in the history of the vegetative nucleus. Such distinctive features occurring at so critical a period as that immediately preceding the appearance of the reduced number of chromosomes cannot be without meaning. They certainly do not usher in a transverse division of chromosomes. It is difficult to believe that so long a preparation is needed in order to accomplish the mere reduction in number. For during the second karyokinesis in the embryosac, which follows on the first almost without interval, the chalazal nucleus exhibits from twenty-four to thirty-two chromosomes in its nuclear plate, though formed itself from the twelve chromosomes of the first division. The true explanation is still to be found.

The question of the identity of the chromosomes throughout 
a series of karyokinetic divisions has been much debated. My observations do not exclude the possibility of such identity. If the network of the resting nucleus be constructed of a single much convoluted filament, and if this filament possess all the properties characteristic of a chromosome, then the chromosomes may preserve their identity throughout a resting-period. We must consider the substance called amorphous chromatin to be of the nature of a foodstuff. I am more disposed to think that the chromosomes are re-cast during each resting-period, part of their chromatin appearing in the amorphous form for redistribution on the spirem ribbon. With regard to the allied question of the relation between dotted ribbons such as those of the immature chromosome in Fig. I9 $a$, and the homogeneous ribbons of a later stage, there is little to say. The change takes place suddenly, and no differentiation can be observed in the ribbon afterwards. A dark line can indeed be traced along its edge, but this is probably due to double refraction. There is no evidence to indicate that the identity of the dots is preserved, but it is not impossible that an incomplete fission may correspond to the plane which separated the two rows of dots.

In describing the phenomena of synapsis I have traced a connexion between the nucleolus and the linin both of the spirem ribbon and of the immature chromosomes. Drops of nucleolar matter are frequent in the cytoplasm during the early stages of spindle formation, and Mr. J. E. S. Moore once pointed out to me in a preparation from the second embryo-sac karyokinesis that a number of these small drops or granules were applied to each chromosome in the nuclear plate. I have frequently observed the same thing since in other preparations of that stage. It would certainly suggest that the nucleolus plays some part in the growth of the chromosomes. The history of their formation from the first embryo-sac nucleus shows that they must contain a great deal of linin.

Little has hitherto been said of the achromatic structure of 
the spindle. The chromosomes behave during karyokinesis as if their segments were drawn apart by the spindle-fibres to which they are attached ${ }^{1}$. The spindles themselves when first formed are irregular in shape, sometimes triangular. My observations throw no light on their origin, and I have never been able to trace centrosomes at their poles.

The curious formation of the lower antipodal nuclei by a process of direct or amitotic division is a pretty illustration of the general tendency among the nuclei of degenerating tissues to divide in that way. Mr. H. H. Dixon has found amitotic divisions in the endosperm of Fritillaria imperialis ${ }^{2}$. The vegetative nucleus of the pollen grain goes a step further and does not divide at all.

\section{APPENDIX ON METHODS.}

\section{A. Fixing.}

The ovaries, gathered between I0 A.M. and 2 P.M., were cut, and the cuticle was partly removed at once. They were then suspended in alcoholic Flemming's solution for $1 \frac{1}{2}$ or 2 hours. The solution was made up as follows :-

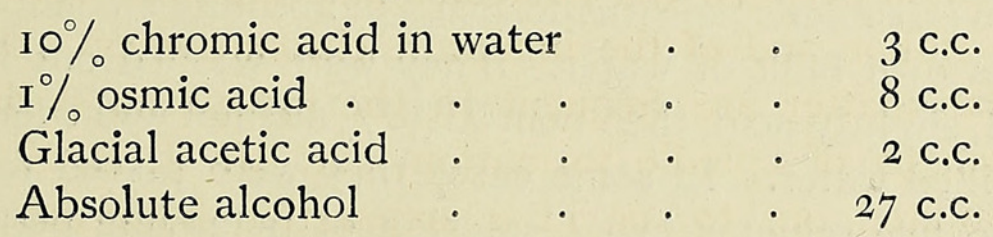

It will only keep for a few hours in the dark. The ovaries were then removed to $5 \%$ aqueous solution of chromic acid for eighteen to twenty-four hours. This gives them a good

\footnotetext{
1 In a communication to the Royal Microscopical Society (I895) I have expressed a different opinion concerning the first division of the pollen mother-cell nucleus. I do not now think that the contorted appearance of the chromosomes implies automatic movement on their part, but rather that it is due to the untwisting of the segments during separation.

${ }^{2}$ H. H. Dixon, Proc. Royal Irish Acad., 3rd ser., vol. iii.
} 
consistency for cutting. They were washed and placed successively in $30 \%, 50 \%, 70 \%$ alcohol at intervals of twentyfour hours, and finally removed to methylated spirit. Those needed for immediate embedding were left in spirit for a day or two, the others preserved in a mixture of about equal parts of alcohol, water and glycerin. The transition from water to spirit was always made in the dark to prevent precipitation of chromic acid.

Other ovaries were placed for a couple of days in absolute alcohol, then transferred to methylated spirit for a week, and preserved in the glycerin mixture.

\section{B. Embedding and cutting.}

I have followed Dr. M. Heidenhain's embedding process with bergamot oil as a penetrating agent. It easily goes bad, and then the sections crumble under the knife. Care must be taken to change the oil as soon as it begins to turn yellow and smell rancid. I found paraffin melting at $55^{\circ} \mathrm{C}$. sufficiently hard.

\section{Stainng.}

I. Flemming's orange method for material fixed in Flemming's solution.

The sections were left about thirty hours in $1 \%$ solution of safranin (Grübler's 'spiritus-löslich') in absolute alcohol diluted with its own bulk of water. They were washed out in $50 \%$ alcohol, slightly acid, then neutral $30 \%$ alcohol and transferred to distilled water. In some cases they were placed for from three to five minutes in $1 \%$ solution of potassium permanganate as a mordant, in others transferred at once to $.25 \%$ solution of gentian violet in water. After remaining in the gentian violet for two to four hours they were washed out successively in $2 \%$ aqueous solution of Grübler's 'orange G,' $I \%$ solution of 'orange $G$ ' in $50 \%$ alcohol, and methylated spirit; dehydrated, and cleared in clove oil.

2. Renaut's haematoxylic eosin for alcohol material.

The sections were left all night in a solution of two to three drops orseillin extract diluted with 100 c.c. of water. 
They were then rinsed and placed in a very dilute solution of Renaut's haematoxylic eosin in $\cdot 1 \%$ aqueous solution of potash alum. This is useless as soon as it goes acid: great care was taken that it should be in good condition, violet not red by transmitted light. The sections may be left in twentyfour hours if the solution is sufficiently dilute. They were always washed afterwards in several changes of hard tapwater to keep them alkaline, and the dilute alcohols through which they passed were made up with tap-water. The orseillin stain is washed out slowly by dilute alcohol.

\section{EXPLANATION OF FIGURES IN PLATES XXII AND XXIII.}

Illustrating Miss E. Sargant's paper on the nuclei of Lilium Martagon.

The figures marked $\times 290$ and $\times 5^{8} 5$ were drawn under Zeiss' apochromatic hom. im. objective 2. m.m. focal length, N.A. I.30 with eye-pieces 2 and 4 respectively. Those marked $\times 1000$ and $\times 1050$ were drawn under Zeiss' apochromatic hom. im. objective I.5 m.m. focal length, N.A. I.30, with eye-piece 6 . The figures were outlined with the camera lucida, and their magnification calculated in the usual way by projection of the scale of a stage micrometer on to the paper scale supplied by Zeiss. The difference between the magnifications of those marked $\times 1000$ and $\times 1050$ respectively is due to a difference in the height of the drawing-board on to which the outline was projected.

\section{Plate XXII. Lilium Martagon.}

Figs. I-8. Vegetative nuclei from the integuments of ovules.

Fig. I. Resting nucleus. $\times$ I000.

Fig. 2. Nucleus in slender spirem. $\times$ I000.

Fig. 3. Nucleus just after segmentation. In this and following figure the chromosomes are ribbon-like. $\times 1000$.

Fig. 4. Early spindle. $\times$ I000.

Fig. 5. Eight chromosomes showing longitudinal fission from an early spindle. $\times 1000$.

Fig. 6. Three chromosomes from a nuclear plate. Separation is beginning from one end. $\times$ I000.

Fig. 7. Chromosomes from a late nuclear plate. $\times 1000$.

Fig. 8. Late diaster. 
Fig. 9. Nucleus from young nucellus.

Fig. 9. Nucleus in early spindle from hypodermal cell occupying a median position in a young nucellus and therefore a possible ancestor for the embryo-sac. $\times$ I000.

Figs. IO-I $2 a$. Nuclei from embryo-sac.

Fig. Io. Median optical section of resting primary nucleus. $\times 5^{8} 5$.

Fig. Io $a$. Tangential view of same. The chromatic thread shows a single row of dots. $\times 1050$.

Fig. II. Later resting stage. $n$ nucleolus. $\times 5^{8} 5$.

Fig. I $a$. Part of chromatic thread (x, fig. I I), more highly magnified. It still shows a single row of dots.

Fig. I 2. Transition to synapsis. The thread has thickened and the dots are larger. $\times 5^{8} 5$.

Fig. I 2 a. Part of chromatic thread more highly magnified. The thickened thread shows a double rows of dots. $n n$ lumps of nucleolar matter.

\section{Plate XXIII. Lilium Martagon.}

Figs. I3-43. Nuclei from embryo-sac.

Fig. I3. Primary nucleus in synapsis. Chromatic thread contracted. Nucleolus sickle-shaped. Membrane obscure. $\times 5^{8} 5$.

Fig. I $3 a$. Part of thread from younger nucleus during synapsis more highly magnified. It is hardly broader than that shown in Fig. $12 a$.

Fig. I3 6. Part of thread from tangential section of nucleus shown in Fig. I3, magnified to same extent as Fig. I3 $a$. It is broader than that of Fig. I3 $a$, but the dots are no larger.

Fig. I4. Primary nucleus in spirem. $\times 5^{8} 5$.

Fig. I 5. Similar nucleus rather later. The chromatic ribbon begins to split longitudinally while still continuous. $\times 5^{8}$.

Fig. I6. Similar nucleus just after segmentation. $\times 585$.

Fig. 17. Primary nucleus soon after segmentation. The long double segments show a tendency to cluster round the washy nucleolus, and the membrane becomes obscure. $\times 5^{8} 5$.

Fig. I8. Primary nucleus a little later. The long segments have contracted and cluster round half-dissolved nucleolus. Each semi-segment shows signs of longitudinal fission. The membrane is obscure. $\times 585$.

Fig. I9. Primary nucleus at stage a good deal later than that shown in Fig. 18. Median section of series. Each immature chromosome consists of two ribbon-like segments which are bordered on either margin by a row of chromatic granules. Membrane broken, perhaps accidentally. The chromosomes are clustered round the nucleolus, which now has a definite outline. $\times 5^{8} 5$.

Fig. I9 $a$. Single immature chromosome at stage corresponding to that of Fig. I9, but from a tangential section of another nucleus. $\times 1050$.

Fig. 20. Primary nucleus with mature chromosomes just before disappearance of nuclear membrane. Traces of the cluster round the nucleolus persist. $\times 5^{8} 5$.

Fig. 2I. First karyokinesis. Five chromosomes from early spindle. Drops of nucleolar matter scattered over spindle and cytoplasm. $\times 5^{8} 5$.

Fig. $22(a-c)$. Three successive sections of a single nuclear plate (first karyokinesis). There are unmistakably twelve chromosomes. $\quad \times 5^{8} 5$. 
Fig. 23. First karyokinesis. Segments of three chromosomes are almost separated. $\times 5^{8} 5$.

Fig. 24. Diaster of first karyokinesis. The upper and lower segments belong to different chromosomes. $\times 585$.

Fig. 25. Dispirem of first karyokinesis. Two rows of dots in chromatic thread. Reconstruction of nucleoli is taking place. $\times 290$.

Fig. 26. Embryo-sac with two resting nuclei. $\times 290$.

Fig. 27. Embryo-sac with two nuclei in early spirem stage. Membrane and nucleoli intact. $\times 290$.

Fig. 28. Embryo-sac with two nuclei in late spirem. Membrane has vanished: traces of nucleoli remain. $\times 290$.

Fig. 29. Second karyokinesis. Micropylar nucleus during segmentation. $\quad \times 5^{8} 5$.

Fig. 3o. Second karyokinesis. Micropylar nucleus has formed nuclear plate. Seven chromosomes are shown in this section which cuts the spindle rather obliquely. The chromosome marked $x$ is dividing from the middle. $\times 5^{8} 5$.

Fig. 3I. Second karyokinesis. Chromosomes from late nuclear plate of micropylar nucleus. $\times 585$.

Fig. 32. Embryo-sac with two nuclei in early dispirem. Chalazal pair larger than micropylar pair. $\times 290$.

Fig. 33. Embryo-sac with two nuclei in late dispirem. Both pairs the same size. $\times 290$.

Fig. 34. Embryo-sac with four resting nuclei of equal size. $\quad \times 290$.

Fig. 35. Embryo-sac with four similar nuclei in early spirem. $\times 290$.

Fig. 36. Embryo-sac with four similar nuclei in late spirem. $\times 290$.

Fig. 37. Embryo-sac with four nuclei differentiated in pairs. $\times 290$.

Fig. 38. Embryo-sac with three upper nuclei in slender spirem. Lowest nucleus resting. $\times 290$.

Fig. 39. Chalazal end of embryo-sac. Upper nucleus has formed early spindle : lower one has begun to divide amitotically. $n$ nucleolus. $\times 290$.

Fig. 40. Chalazal end of embryo-sac. Upper nucleus still in early spindle stage but later than that of Fig. 39: lower nucleus constricted. $\times 290$.

Fig. 4r. Chalazal end of embryo-sac. Upper nucleus in early dispirem. Lower nucleus in pseudo-dispirem. $\times 290^{\circ}$.

Fig. 42. Third karyokinesis. Six chromosomes from nuclear plate of micropylar nucleus. $\times 585$.

Fig. 43. Third karyokinesis. Three chromosomes from late nuclear plate of lower micropylar nucleus. $\times 5^{8} 5$. 


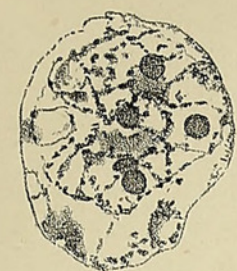

1.

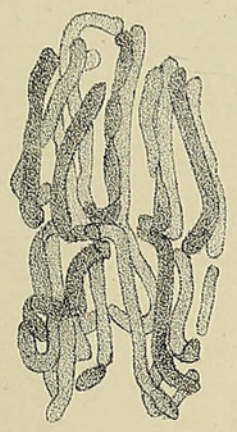

4.

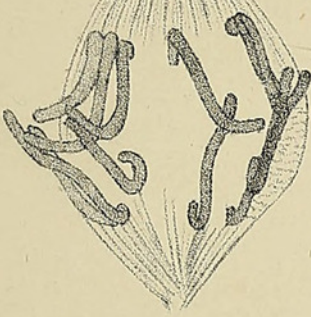

7.

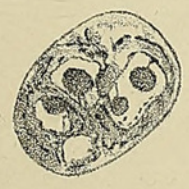

10.

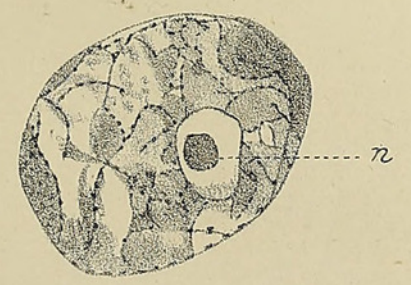

$10^{a}$

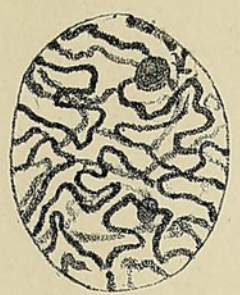

2.

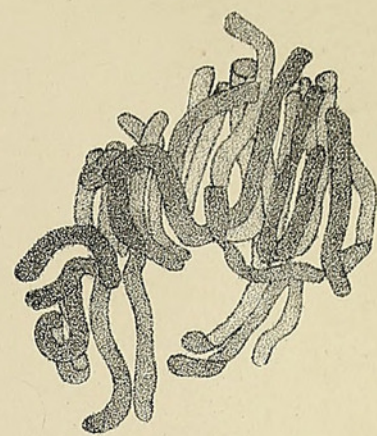

3.

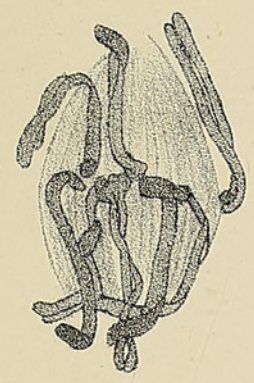

5.

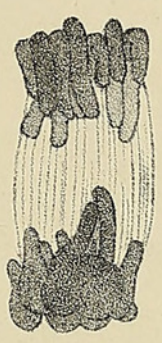

8.<smiles>[CH]1C[C]2C[C]1C2</smiles>

$11^{a}$

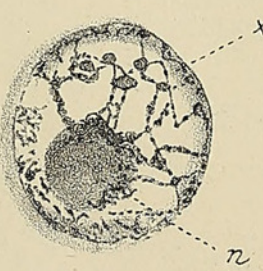

11 .
12 a

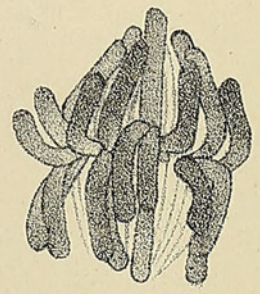

9.
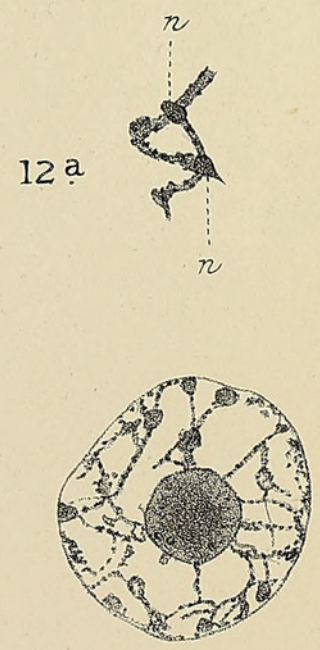

12.

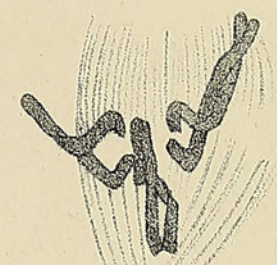

6.

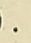

in

University Press, Oxford.

E. S. del. 
Annals of Botany
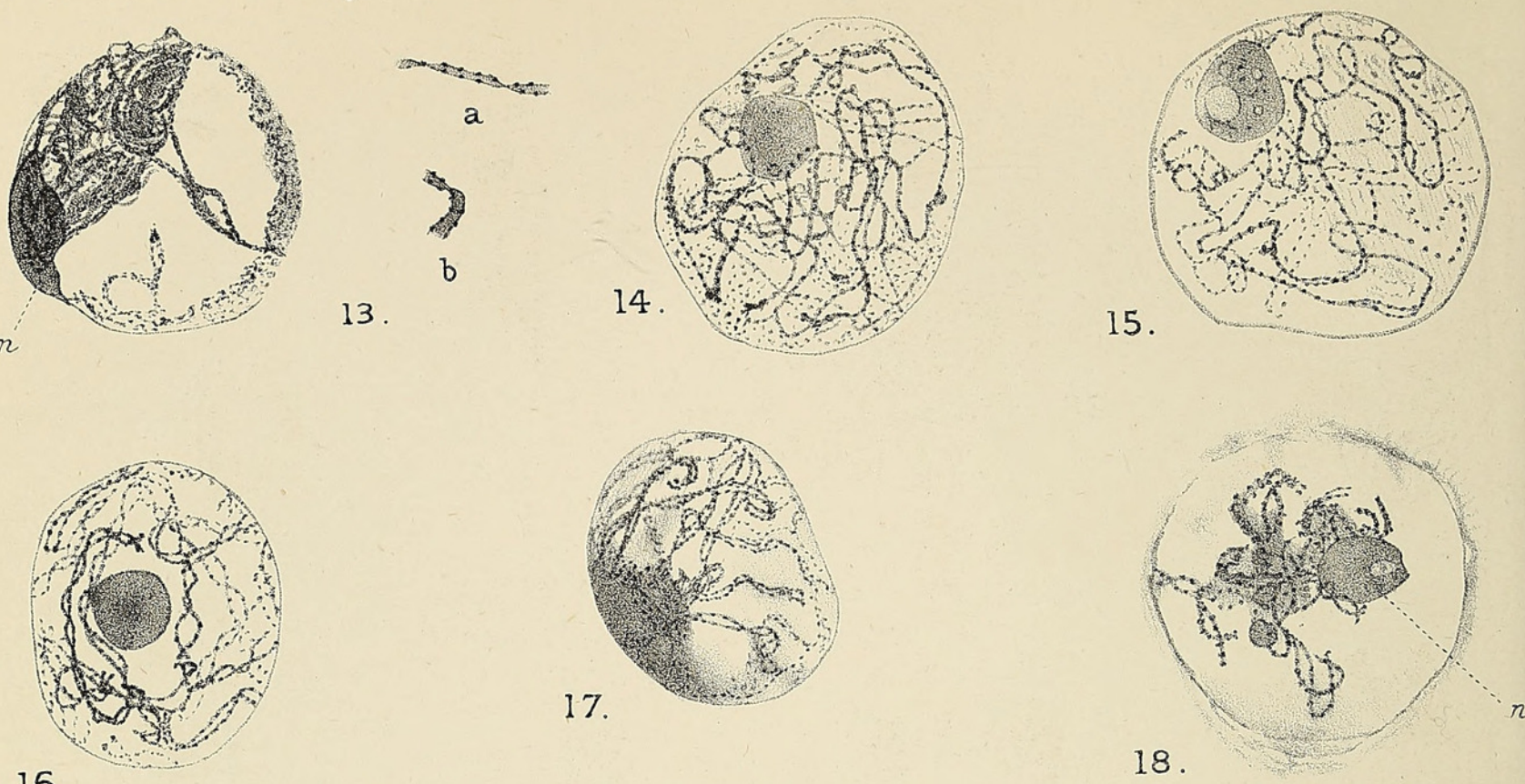

16.

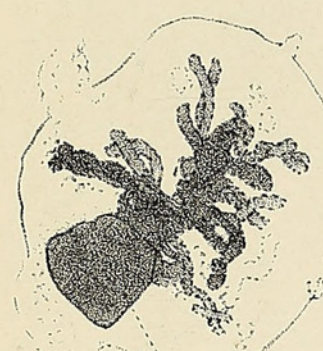

19.

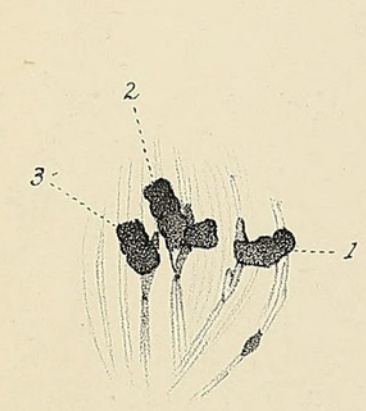

a

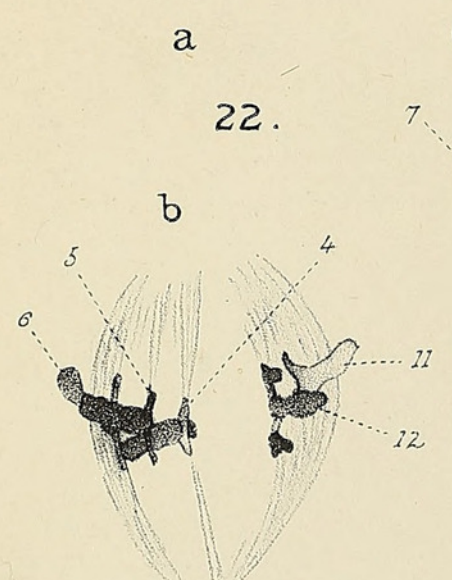

E. S. de?
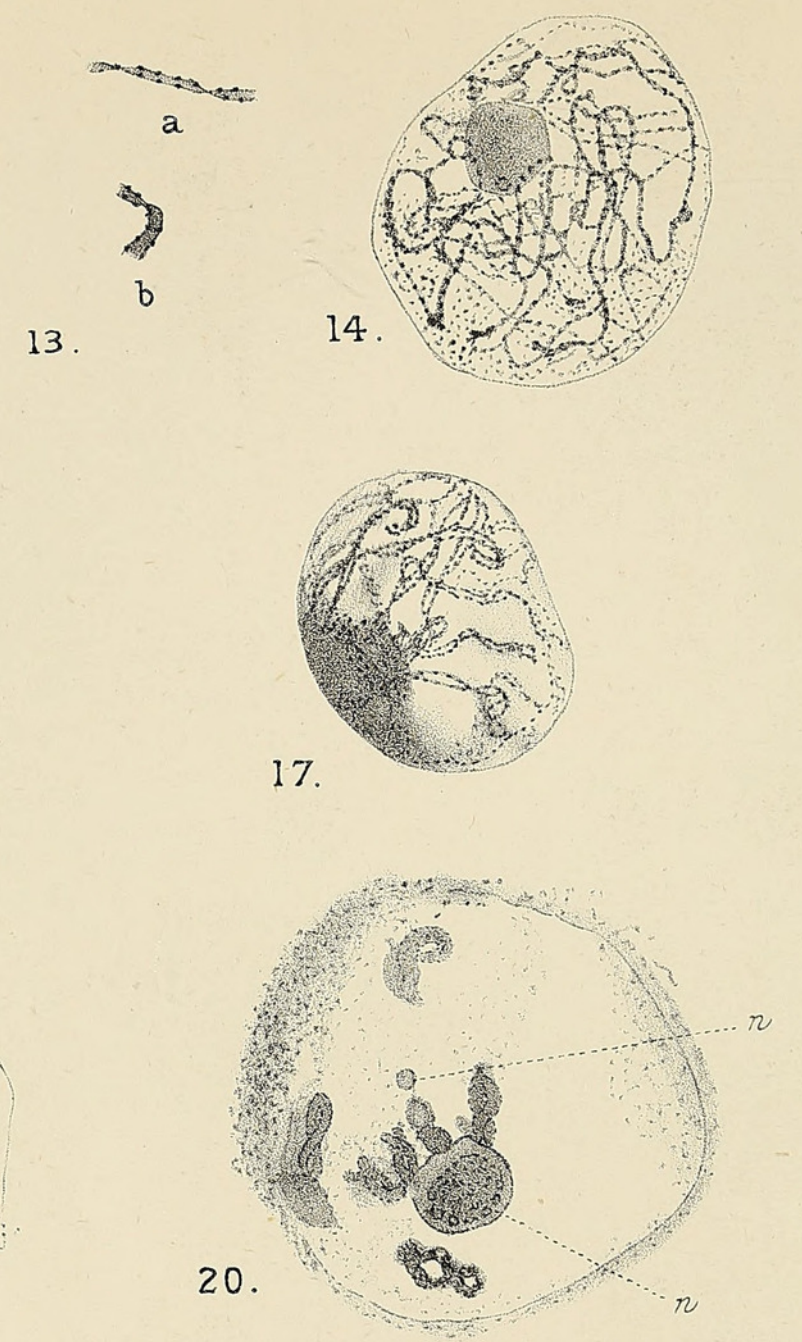

17
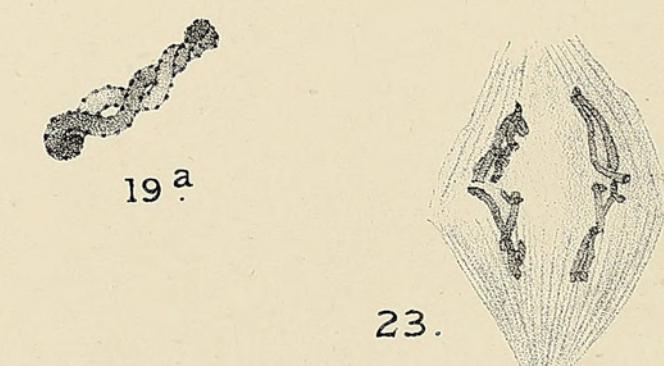

23.

c
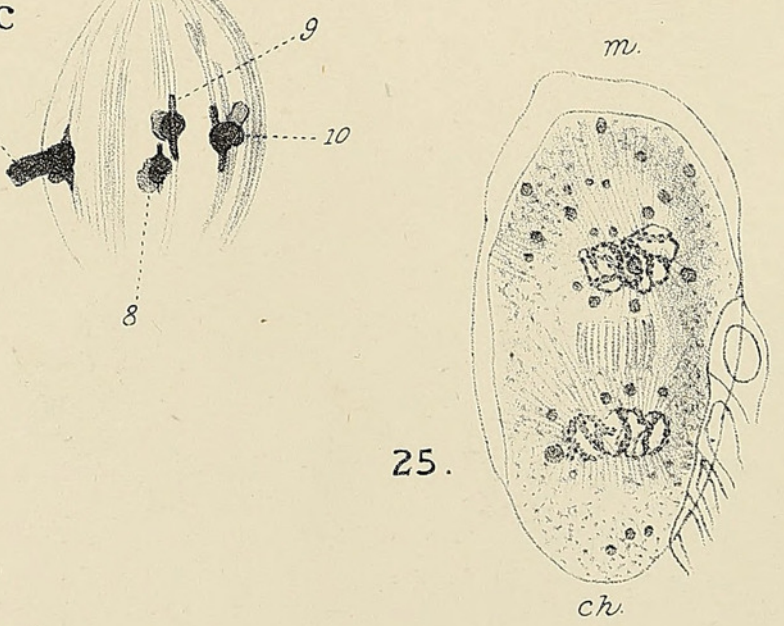

21.
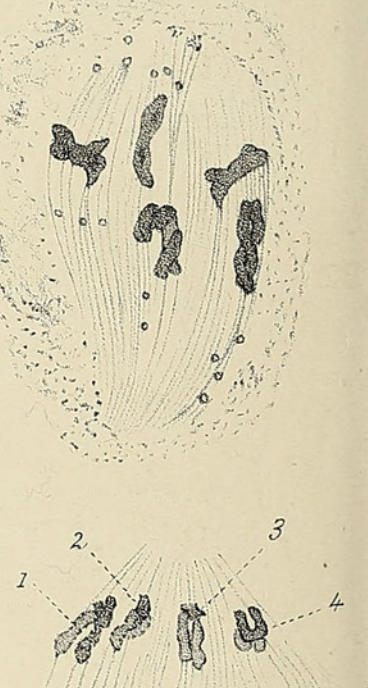

24.
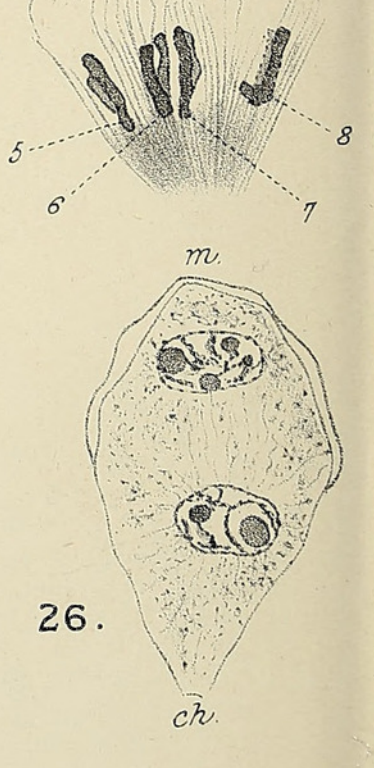


\section{$2 \mathrm{BHL}$ Biodiversity Heritage Library}

Sargant, Ethel. 1896. "The formation of the sexual nuclei in Lilium martagon." Annals of botany 10, 445-477. https://doi.org/10.1093/oxfordjournals.aob.a088622.

View This Item Online: https://www.biodiversitylibrary.org/item/235772

DOI: https://doi.org/10.1093/oxfordjournals.aob.a088622

Permalink: https://www.biodiversitylibrary.org/partpdf/318434

\section{Holding Institution}

Smithsonian Libraries

\section{Sponsored by}

Biodiversity Heritage Library

\section{Copyright \& Reuse}

Copyright Status: Not in copyright. The BHL knows of no copyright restrictions on this item.

This document was created from content at the Biodiversity Heritage Library, the world's largest open access digital library for biodiversity literature and archives. Visit BHL at https://www.biodiversitylibrary.org. 